\title{
Microfibrillated lignocellulose (MFLC) and nanopaper films from unbleached kraft softwood pulp
}

\author{
Erfan Oliaei $(\mathbb{D} \cdot$ Pär A. Lindén $(\mathbb{D} \cdot$ Q Qiong Wu • Fredrik Berthold • \\ Lars Berglund (i) $\cdot$ Tom Lindström (1)
}

Received: 27 August 2019/Accepted: 11 December 2019/Published online: 28 December 2019

(C) The Author(s) 2019

\begin{abstract}
Microfibrillated cellulose (MFC) is an important industrial nanocellulose product and material component. New MFC grades can widen the materials property range and improve product tailoring. Microfibrillated lignocellulose (MFLC) is investigated, with the hypothesis that there is an optimum in lignin content of unbleached wood pulp fibre with respect to nanofibril yield. A series of kraft fibres with falling Kappa numbers (lower lignin content) was prepared. Fibres were beaten and fibrillated into MFLC by high-pressure microfluidization. Nanosized fractions of fibrils were separated using centrifugation. Lignin content and carbohydrate analysis, total charge, FE-SEM, TEM microscopy and suspension rheology characterization were carried out. Fibres
\end{abstract}

with Kappa number 65 (11\% lignin) combined high lignin content with ease of fibrillation. This confirms an optimum in nanofibril yield as a function of lignin content, and mechanisms are discussed. MFLC from these fibres contained a 40-60 wt $\%$ fraction of nanosized fibrils with widths in the range of $2.5-70 \mathrm{~nm}$. Despite the large size distribution, data for modulus and tensile strength of MFLC films with $11 \%$ lignin were as high as $14 \mathrm{GPa}$ and $240 \mathrm{MPa}$. MFLC films showed improved water contact angle of $84-88^{\circ}$, compared to neat MFC films $\left(<50^{\circ}\right)$. All MFLC films showed substantial optical transmittance, and the fraction of haze scattering strongly correlated with defect content in the form of coarse fibrils.

E. Oliaei · F. Berthold · T. Lindström ( $₫)$

RISE Bioeconomy, SE-11486 Stockholm, Sweden

e-mail: Toml@kth.se

E. Oliaei

e-mail: erfan.oliaei@ri.se

E. Oliaei · P. A. Lindén · Q. Wu · L. Berglund ( $₫)$

Department of Fiber and Polymer Technology,

Wallenberg Wood Science Center, KTH Royal Institute of

Technology, SE-10044 Stockholm, Sweden

e-mail: Blund@kth.se

P. A. Lindén

e-mail: parlinde@kth.se 


\section{Graphic abstract}

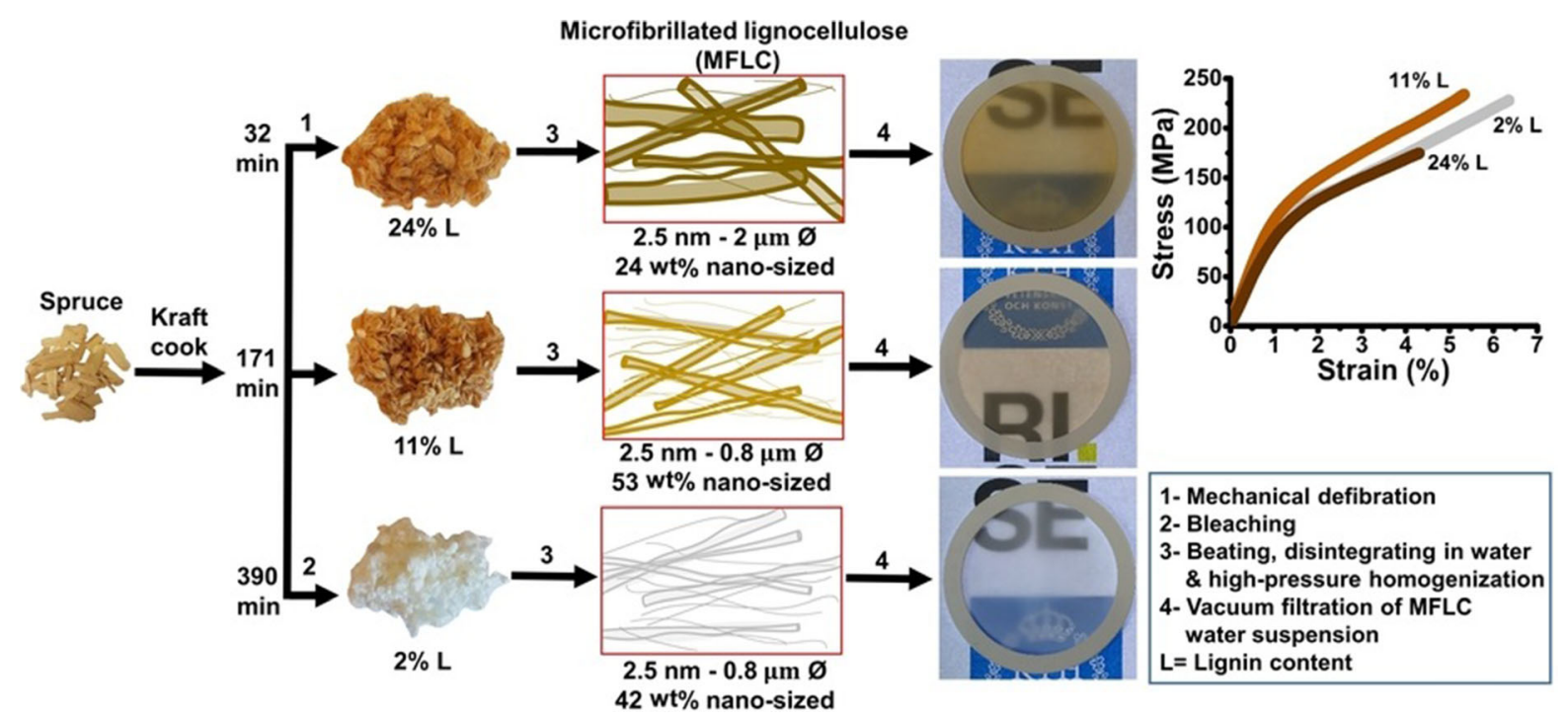

Keywords Lignin-containing cellulose nanofibril $($ LCNF) · Nanocellulose · Lignin · Fibrillation ·

Mechanical properties

\section{Introduction}

In the late 1970s, cellulose fibres were for the first time fibrillated in a process where pulp fibres were passed through a homogeniser (Herrick et al. 1983; Turbak et al. 1983). Since these pioneering efforts, microfibrillated cellulose (MFC) has recently been developed into industrial products. Due to the interesting characteristics of fibrillated nanocellulose, e.g. large aspect ratio and high mechanical properties (Isogai et al. 2011; Klemm et al. 2011; Moon et al. 2011; Nishiyama 2009), this type of material component is used or considered for applications in paper and paperboard, coatings, adhesives, biocomposites (Berglund and Peijs 2011; Dufresne 2018), oil recovery, viscosity modifiers, cement applications, water remediation technologies, aerogels/foams, electroactive materials and medical and pharmaceutics applications (Lindström et al. 2014).

Over the years, many methods were developed for fibrillation of cellulosic plant fibres including microfluidization, extrusion, grinding/refining, milling, cryo-crushing, aqueous counter collision and ultrasonication (Klemm et al. 2011; Moon et al. 2011). Fibrillation of wood cellulose fibres is energy-intensive but can be facilitated by pre-treatment such as induction of electrostatically charged groups, mechanical treatment or mild acid or enzymatic treatment (Klemm et al. 2018; Moon et al. 2011). Electrostatically charged groups can be introduced to fibril surfaces in the wood pulp fibre cell wall during pulping and bleaching operations or later by carbonyl or carboxyl groups through e.g. TEMPO (2,2,6,6tetramethylpiperidinyloxyl) catalyzed oxidation (Atalla and Isogai 2010; Isogai et al. 2011) or through e.g. carboxymethylation (Klemm et al. 2018; Wågberg et al. 2008), or cationic functionalities (Olszewska Eronen et al. 2011).

Since the development of MFC, most studies have been carried out on MFC prepared from bleached pulp fibres of very low lignin content. Microfibrillated lignocellulose (MFLC) has attracted little attention. However, MFLC is interesting since it shows higher electronegative charge, higher yield and the chemical characteristics are different from those of bleached kraft MFC. Native wood contains very few carboxyl groups (Sjöström 1989), but during pulping hemicelluloses are deacetylated (Katz et al. 1981). New carboxyl groups are also introduced to lignin by alkaline oxidation or disproportionation reactions of the Cannizzaro type (Sarkanen and Ludwig 1971). Therefore, unbleached fibres have higher charge 
density (Gellerstedt and Lindfors 1987; Sjöström 1989), which may facilitate the fibrillation process.

Lignin has antioxidant function which stabilizes cellulosic mechano-radical formation during fibrillation and reduces chemical crosslinking in the fibre cell wall (Rojo et al. 2015). MFLC from wood is interesting for packaging (Spence et al. 2011) and composite manufacturing since lignin reduces moisture sorption (Jiang et al. 2019; Rojo et al. 2015), may impair $\mathrm{O}_{2}$ transmission (Rojo et al. 2015), and may flow during heating (Abe et al. 2009). The presence of lignin can for specific processing conditions lead to reduced porosity in nanocellulose films (Herrera et al. 2018; Jiang et al. 2019; Rojo et al. 2015). MFLC shows lower hydrophilicity, amphoteric (acid and base) character (Herrera et al. 2018; Rojo et al. 2015; Wei et al. 2018), and improved compatibility with hydrophobic polymers (Herzele et al. 2016; Rojo et al. 2015; Wei et al. 2018).

Previous MFLC studies are commonly either based on a specific wood starting material (i.e. wood flour or specific pulp fibres) (Abe et al. 2009; Herzele et al. 2016; Osong et al. 2013; Rojo et al. 2015; Yousefi et al. 2013), specific chemical treatments (Herrera et al. 2018; Herzele et al. 2016) or non-wood biomass (Jiang et al. 2019; Lu et al. 2018; Peng et al. 2018; Yousefi et al. 2018). Here, the focus is on commercially available unbleached Kraft fibres as a source, and the investigation is systematic on effects of processing and lignin content on structure and composition of the corresponding MFLC grades.

Lignin has many advantages mentioned in the previous section, but is also intermixed in the fibre cell wall and functions as a binder for cellulose fibrils, so that mechanical fibrillation is impeded. The main hypothesis investigated is therefore that unbleached kraft pulp subjected to different pulping conditions shows an optimum lignin content for high MFLC fibrillation yield. The MFLC is thoroughly characterized and used for nanopaper structures, which are evaluated with respect to material properties. The present MFLC is of high technical relevance due to the use of unbleached kraft fibres readily available in industry. It will also be demonstrated that MFLC can show excellent mechanical film properties, without removal of large-sized cell wall fragments.

\section{Materials and methods}

Kraft pulping protocol

Classical Kraft pulping was applied to hand-sorted spruce wood chips (with 91\% dryness) using an effective alkali of $18 \%$, a sulfidity of $20 \%$ and a liquor to wood ratio of $4: 1$ at $160{ }^{\circ} \mathrm{C}$. After 10 min temperature equilibration, samples (each 250-500 g) were cooked for $32 \mathrm{~min}, 90 \mathrm{~min}, 171 \mathrm{~min}, 312 \mathrm{~min}$ and $390 \mathrm{~min}$, corresponding to total H-factors of 64, 201, 390, 722 and 906, respectively. These conditions yielded a series of pulp fibres with a wide range of Kappa numbers (lignin content).

Kraft pulp fibre preparation

The samples cooked for $32 \mathrm{~min}$ and $90 \mathrm{~min}$ were defibrated from wood tissue using a Sprout-Waldron refiner. The sample cooked for $171 \mathrm{~min}$ was readily defibrated after 10,000 revolutions using a pulp disintegrator. The rest of the samples did not need mechanical defibration. However, the fibres of all samples were liberated using water jets and the rejects were separated over a Wennberg screen with $0.2 \mathrm{~mm}$ thick slots (AB Lorentzen \& Wettre, Sweden). Afterwards, the pulp fibres were dewatered to a concentration of about $30 \mathrm{wt} \%$, using a centrifuge, and were then comminuted to a fluffy form.

\section{Bleaching}

A fraction of the sample cooked for 390 min was bleached using an oxygen delignification step, and four consecutive chlorine dioxide bleaching steps. Oxygen delignification was performed using an alkali charge of $2.5 \mathrm{wt} \%$ and a pulp concentration of $10 \mathrm{wt} \%$ at an applied oxygen pressure of 8 bar at $100{ }^{\circ} \mathrm{C}$ for $30 \mathrm{~min}$. The initial two chlorine dioxide steps were performed using a chemical charge equal to Kappa number $\times 0.2 \mathrm{wt} \%$ (i.e. $4 \mathrm{wt} \%$ and $2.8 \mathrm{wt} \%$, respectively) and a pulp concentration of $10 \mathrm{wt} \%$, adjusted to $\mathrm{pH} 4$ with acetic acid, at $80{ }^{\circ} \mathrm{C}$ for $2 \mathrm{~h}$. The third chlorine dioxide step was done using a fixed chemical charge of $1 \mathrm{wt} \%$, while the fourth was done using a fixed chemical charge of $0.5 \mathrm{wt} \%$. Otherwise, the same parameters were used for the first and second steps. 
Alkaline washing

In order to transfer the pulps into the $\mathrm{Na}^{+}$form, the following procedure was adopted from the Lindström et al. study (Lindström and Carlsson 1982) with slight changes:

(a) Washing with deionized water for 5 times

(b) Immersing in an aqueous solution of $10 \mathrm{mM}$ $\mathrm{HCl}(\mathrm{pH} \simeq 2)$ for $1 \mathrm{~h}$

(c) Washing with deionized water for 5 times

(d) Immersing in an aqueous solution of $4 \mathrm{mM}$ $\mathrm{Na}_{2} \mathrm{CO}_{3}(\mathrm{pH} \simeq 10.6)$ for $1 \mathrm{~h}$

(e) Washing with deionized water for 5 times

Each of the above steps started with a fibre suspension of about $1 \%$ concentration, followed by Büchner funnel filtration to a concentration of about $20 \%$, before next dilution. The transformation of the pulp fibres into their sodium form is essential for swelling, as it reduces the fibres to their ionic form and provides sodium counter-ion charge (Lindström 1992; Lindström and Carlsson 1982). All experiments were performed in $\mathrm{Na}^{+}$form of the pulp fibres, except the total charge measurement, which was performed on protonated fibres.

Kappa number

Kappa numbers were measured in accordance with ISO 302:2015.

Chemical composition

The extractives content was determined via solvent extraction in a Soxhlet extraction apparatus first for $12 \mathrm{~h}$ in water and then for $12 \mathrm{~h}$ in ethanol according to ASTM E1690 - 08.

The Klason lignin content was measured according to the standard TAPPI T222 om-02, and sugar analysis was carried out after acid hydrolysis, using a highperformance anion-exchange chromatography Dionex ICS-3000 (Thermo Fisher Scientific Inc., USA) with references of rhamnose, arabinose, galactose, glucose, xylose, and mannose (Herrera et al. 2018). The hemicellulose and cellulose contents were calculated based on glucomannan and xylan as the principal hemicelluloses in spruce (Yang et al. 2018). Xylan content was calculated based on xylose and arabinose, glucomannan content was calculated based on galactose, glucose, and mannose contents using a 1:3 ratio of glucose: mannose (Sjöström 1993; Walker 2006; Yang et al. 2018), and cellulose content was then calculated based on the remaining glucose content.

Total charge measurement

The total charge of pulp fibres correlates with carboxylic acid content, and was measured by conductometric titration according to SCAN-CM 65:02, using an automated Metrohm titrator (Titrino $702 \mathrm{SM}$ Metrohm AG, Herisau, Switzerland).

PFI milling

21 of deionized water was added to $30 \mathrm{~g}$ of each pulp sample and disintegrated for 30000 revolutions in a pulp disintegrator and then filtered to a concentration of $10 \mathrm{wt} \%$. The pulp fibres were then beaten in a laboratory PFI mill at $10 \mathrm{wt} \%$ concentration and a load of $33.3 \mathrm{~N}$ according to ISO 5264-2:2011. The samples were beaten 3 times each for 10,000 revolutions with 15 min interval between each step to let the pulp fibres cool down.

Water retention value

Water retention value (WRV) measurements were carried out in accordance with SCAN-C 62-00:2000.

Homogenisation

Aqueous suspensions of beaten pulps at a concentration of about $0.25 \mathrm{wt} \%$ and with a $\mathrm{pH}$ around 7.5 were homogenised using a high-pressure microfluidizer (Microfluidizer M-110EH, Microfluidics Corp., USA). The homogenisation process was conducted employing two serial coupled Z-shaped interaction chambers with path diameters of 400 and $200 \mu \mathrm{m}$ at a pressure of about 600 bar for 4 passes, followed by employing two serial coupled Z-shaped interaction chambers with path diameters of 200 and $100 \mu \mathrm{m}$ at a pressure of about 1500 bar for 5 passes. 
Preparation and characterization of nanopaper films

Nanopaper structures based on 3 types of the prepared MFLC:s were fabricated by a filtration procedure (Sehaqui et al. 2010). Aqueous suspensions of microfibrillated cellulose at a concentration of $0.1 \mathrm{wt} \%$ were stirred overnight, degassed for $2 \mathrm{~h}$ and then vacuum filtered on membranes with a pore size of $0.65 \mu \mathrm{m}$ (DVPP, Millipore) with a glass filter funnel of $7.2 \mathrm{~cm}$ in diameter. After filtration, the wet films were stacked on filter papers and fixed with frames and then dried at $50{ }^{\circ} \mathrm{C}$ overnight. Conditioning was carried out for $48 \mathrm{~h}$ at $23{ }^{\circ} \mathrm{C}$ and $50 \%$ relative humidity.

The thicknesses of the nanopaper structures were measured using TJT STFI thickness tester M201 and at least 12 points were measured for each sample. The apparent density was calculated according to the measured weight basis and thickness after conditioning. For the tensile test, strips of $6 \mathrm{~mm} \times 45 \mathrm{~mm}$ were punched from the nanopaper and loaded in a universal MTS tensile machine. The tests were conducted with a gauge length of $30 \mathrm{~mm}$ and at a crosshead speed of $5 \mathrm{~mm} / \mathrm{min}$.

The static water contact angle of nanopaper samples (hot-pressed according to (Rojo et al. 2015)) was measured using a contact angle meter CAM20 (KSV Instruments, Finland). A water droplet with a volume of $4 \mu \mathrm{L}$ was placed on the surface and the contact angle was measured after $10 \mathrm{~s}$. The average of at least three measurements is reported.

\section{Microscopy}

A Hitachi TM-1000 Tabletop SEM for was used for imaging of the kraft fibres, freeze-dried on a screen cloth. A Hitachi S-4800 field-emission scanning electron microscope (SEM) was used for imaging and morphological studies of MFLC. Dilute water suspensions of fibrils were sonicated and then filtered through nanoporous aluminium oxide membranes (FlexiPor $20 \mathrm{~nm}$; SmartMembranes GmbH, Halle, Germany) to deposit $1-10 \mathrm{mg} / \mathrm{m}^{2}$ of fibrils on the membranes. Afterwards, the specimens were sputtercoated to 1-2 nm thick platinum-palladium layer using a 208HR Cressington Sputter Coater (Cressington Scientific Instruments, Watford, UK) according to (Larsson et al. 2018).
Transmission Electron Microscopy (TEM, Hitachi HT7700) was performed at $100 \mathrm{kV}$ accelerating voltage. The samples were prepared by adding a droplet of about $0.01 \mathrm{wt} \%$ lignocellulose nanofibril suspension onto ultrathin carbon-coated 400 mesh copper grids (Ted Pella, USA). The excess liquid was removed by a filter paper after 1 min. Afterwards, a drop of about $2 \mathrm{wt} \%$ uranyl acetate negative stain was deposited on the grid and the excess solution was removed after $1 \mathrm{~min}$. The sample was then dried overnight in darkness at ambient conditions. All micrographs were obtained with a charge-coupled device (CCD) camera. Image processing of electron micrographs was done using ImageJ (https://imagej. nih.gov/ij/) and "Microscope Measurement Tools" plugin.

\section{Rheological studies}

The rheological investigations were operated by Kinexus stress-controlled rotational rheometer (Malvern Instruments, UK) and iSpace software (Malvern Instruments, UK). Concentric metal cylinder geometries (bob and cup) with roughened surfaces were used in the studies. The samples were loaded into the device and were equilibrated for $2 \mathrm{~min}$ before the measurements. The measurements were carried out in a range of $0.1-100 \mathrm{~s}^{-1}$ shear rate, $3 \mathrm{~min}$ sampling interval and $20 \mathrm{~s}$ integration time at $25^{\circ} \mathrm{C}$.

Centrifugation and determination of nanofibril yield

Microfibrillated lignocelluloses (MFLC:s) with different size distribution and charge content were centrifuged to separate the nano-sized particles, with carefully tuned centrifugation conditions. Instead of employing a high centrifugal field, which is common for highly charged CNF suspensions, a more cautious centrifugation method was used, since low-charged MFLC:s are inherently unstable under high centrifugal fields.

The centrifugation condition was tuned for sedimentation of large fibrils of MFLC suspensions in $15 \mathrm{~min}$. To control and keep the same sedimentation rate for different MFLC suspensions, centrifugal fields were adjusted for each MFLC, separately. For this reason, centrifugal field was varied for concentrated suspensions $(0.19 \mathrm{wt} \%)$ of every MFLC, so that the 
Table 1 Preparation conditions and results for the pulp fibres

\begin{tabular}{lllllll}
\hline Sample & $\begin{array}{l}\text { Kappa } \\
\text { number }\end{array}$ & $\begin{array}{l}\text { Pulping } \\
\text { time (min) }\end{array}$ & $\begin{array}{l}\text { Total pulping } \\
\text { yield (\%) }\end{array}$ & $\begin{array}{l}\text { Mechanical } \\
\text { defibration }\end{array}$ & $\begin{array}{l}\text { Screening } \\
\text { rejects (\%) }\end{array}$ & Bleaching \\
\hline F-K114 & $113.9(1.3)$ & 32 & 70.4 & Yes & 39.4 & - \\
F-K96 & $95.5(0.9)$ & 90 & 62.1 & Yes & 4.6 & - \\
F-K65 & $65.4(0.4)$ & 171 & 56.5 & - & 1.4 & - \\
F-K34 & $34.3(0.2)$ & 312 & 53.0 & - & 1.1 & - \\
F-K27 & $26.6(0.5)$ & 390 & 51.4 & - & 0.5 & - \\
F-K2 & $1.9(0.1)$ & 390 & $48.8^{\mathrm{a}}$ & - & - & Yes \\
\hline
\end{tabular}

${ }^{\text {a }}$ This value is calculated by consideration of pulping yield and the amount of removed lignin as estimated from composition data after bleaching (see Table 2). Values in parentheses are standard deviations

adjusted centrifugal field is the maximum, where volume of the sediment does not drop below $95 \%$ of the whole. However, for MFLC-K114, it was close, but not above $95 \%$ due to the instrument limitation. Centrifugal fields for MFLC:s with K2, K27, K34, k65, k96 and k114 (see Table 1) were 40, 190, 150, 120,50 and $21 \mathrm{~g}$, respectively. The aim was applying suitable centrifugal force fields to separate finer fibril from larger fibril structures. The difference in suitable centrifugal fields originates from dissimilarities regarding density, morphology, size, electrostatic forces and physical interaction of constituent fibrils (Wilson and Goulding 1986). The critical overlapping concentration was also determined, in order to ensure sufficiently low concentration for efficient fractionation by centrifugation. Below the critical overlapping concentration, separation occurs readily (Naderi et al. 2014). Based on shear flow of the MFLC suspensions, shear stress versus concentration at different shear rates was determined, showing the occurrence of overlapping in the range of 0.04-0.06 wt\%. Centrifugation of the MFLC suspensions was applied at 0.02 $\mathrm{wt} \%$, which was below the critical overlapping concentration.

About $750 \mathrm{~g}$ of each well-agitated freshly-made MFLC suspension was centrifuged. The centrifuged samples were stabilized for a few minutes and the top $70 \%$ was taken as the supernatant. The concentration of MFLC:s and their supernatant were calculated using filtration and drying of suspensions on a membrane with a pore size of $0.65 \mu \mathrm{m}$ (DVPP, Millipore). At last, the ratio of supernatant to the precentrifugation concentration multiplied by supernatant volume fraction-fibrillation yield-was determined. SEM and TEM of the supernatant proved the separation of nano-sized fibrils from the larger ones.

\section{Fractionation}

The fractionation method was carried out for the purpose of microscopy characterization only, basically according to (Larsson et al. 2018), with a difference in centrifugation condition. As illustrated in Fig. 1, the first two steps of fractionation were performed in a Britt Dynamic Drainage Jar equipped with a vaned jar and two metal sieves with pore sizes of $20 \mu \mathrm{m}$ and $1-2 \mu \mathrm{m}$ at $3000 \mathrm{rpm}$ stirring rate. The process was started by adding 0.51 of MFLC at a concentration of $0.2 \mathrm{wt} \%$ and then 21 of deionized water was gradually added. The filtrate was then set aside, and the retentate was rinsed with 51 of deionized water to remove residual amounts of smaller fibre fractions. About 0.11 of retentate was collected as the coarse fraction (F1). The filtrate from the first fractionation step was then filtered using the finer sieve with the above-mentioned procedure plus adding 51 of the rinse water from the previous step. This procedure yields about 0.11 of retentate as the second coarse fraction (F2), a filtrate of about 4.31 and removal of 101 of rinse water with a loss of small particles. Therefore, concentration of the final filtrate falls below $0.023 \mathrm{wt} \%$, which is low enough for centrifugation of the prepared MFLC suspensions (see the result and discussion part). Finally, the centrifugation was done at the same conditions of nanofibril yield determination, resulting in the sediment as the 


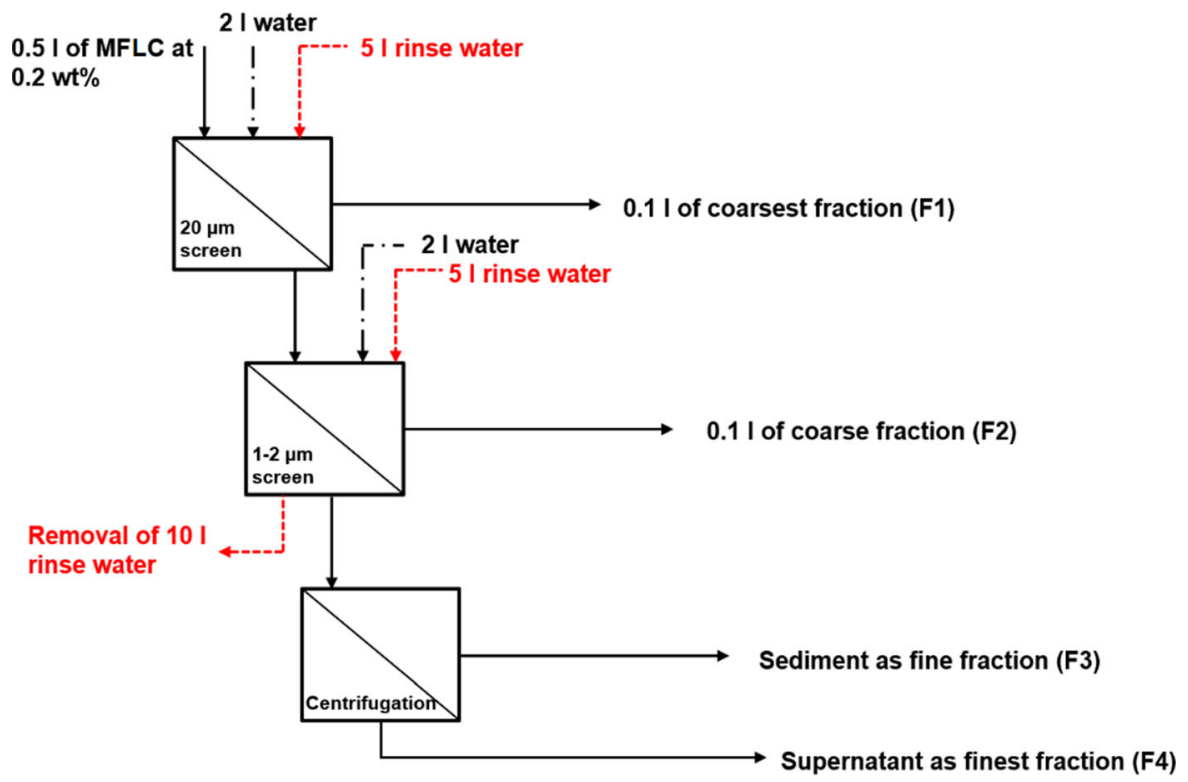

Fig. 1 Schematic fractionation procedure of an MFLC suspension (recreated based on reference Larsson et al. (2018))

Table 2 Relative chemical composition of spruce and kraft pulp fibres

${ }^{\mathrm{a} K}$ Klason lignin
${ }^{\mathrm{b}}$ Extractives content of
F-K34, F-K27 and F-K2
were not analysed and
assumed to be zero to
calculate the other
compositions

fine fraction (F3) and the supernatant as the finest fraction $(\mathrm{F} 4)$.

\section{Results and discussion}

Spruce wood chips were subjected to kraft pulping so that unbleached pulp fibres were obtained. Fibres with different lignin content were prepared by controlling pulping conditions (see Table 1). Kraft fibres with Kappa number 114 (23.6\% lignin) were straight and rod-like (Fig. 2). Fibres with Kappa number 65 did not collapse and the original structure was well-preserved, although some kinks were apparent. The fibres with Kappa number 2 (1.8\% lignin) were partially collapsed and showed comparatively more kinks and curved appearance due to the longer history of chemical treatment.

Optimum conditions were sought so that the resulting fibres could be readily disintegrated into microfibrillated lignocellulose (MFLC). High lignin content provides desirable charge but also impedes fibrillation since lignin functions as a binder for the cellulose fibrils in the fibre cell wall.

A longer pulping time resulted in a lower Kappa number, see Table 1, corresponding to lower lignin content. The amount of screening reject was also lowered with pulping time. In Table 1, the samples were named after their corresponding Kappa number. The fibres were coded as "F", homogenised microfibrillated lignocellulose suspensions as "MFLC", and 

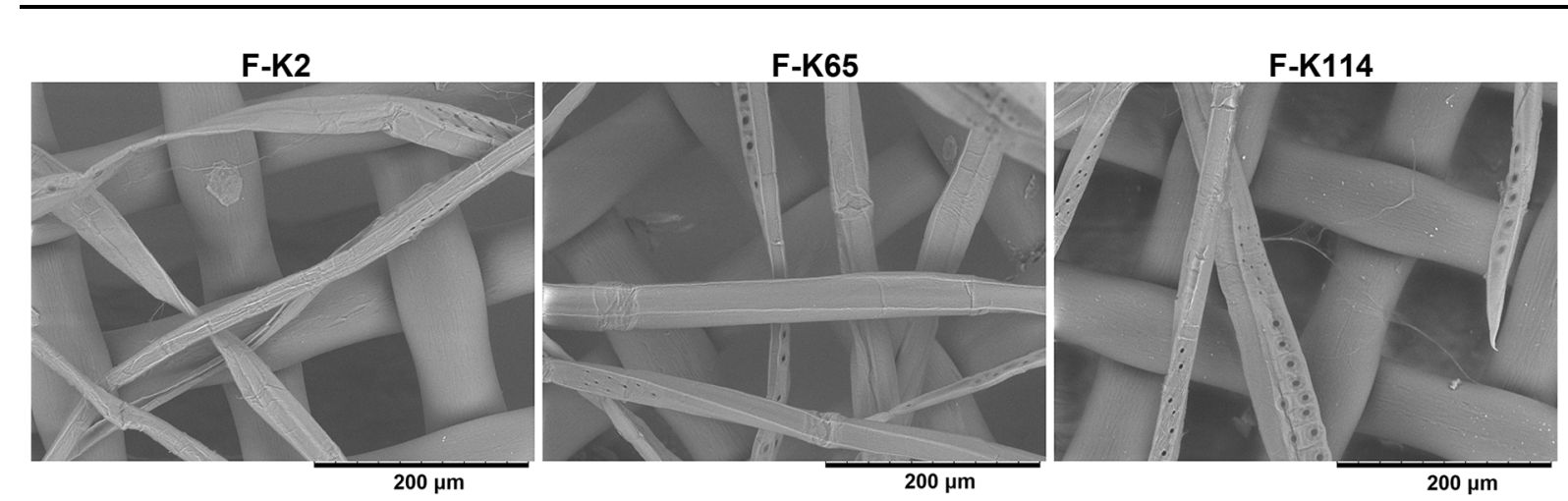

Fig. 2 SEM images of F-K2, F-K65 and F-K114, freeze-dried on a screen cloth (K2, K65 and K114 refer to Kappa number, see Table 1)

the lignocellulose nanofibril supernatant of centrifuged MFLC as "LCNF", respectively.

The Klason lignin content is reported in Table 2. The extractives content in the fibres was limited. Furthermore, there was no significant difference in hemicellulose content of fibres, but the samples with lower amount of lignin obviously showed higher cellulose content.

The total charge content of fibres was measured and plotted against the Kappa number in Fig. 3. The lowest total charge, about $40 \mu \mathrm{equ} / \mathrm{g}$, was associated with the bleached sample (F-K2) and the highest charge, about $96 \mu \mathrm{equ} / \mathrm{g}$, was associated with the highest lignin content and Kappa number (F-K114). There was an almost linear relationship between the Kappa number and total charge density of fibres (Fig. 3). The reason is that carboxyl groups in xylan

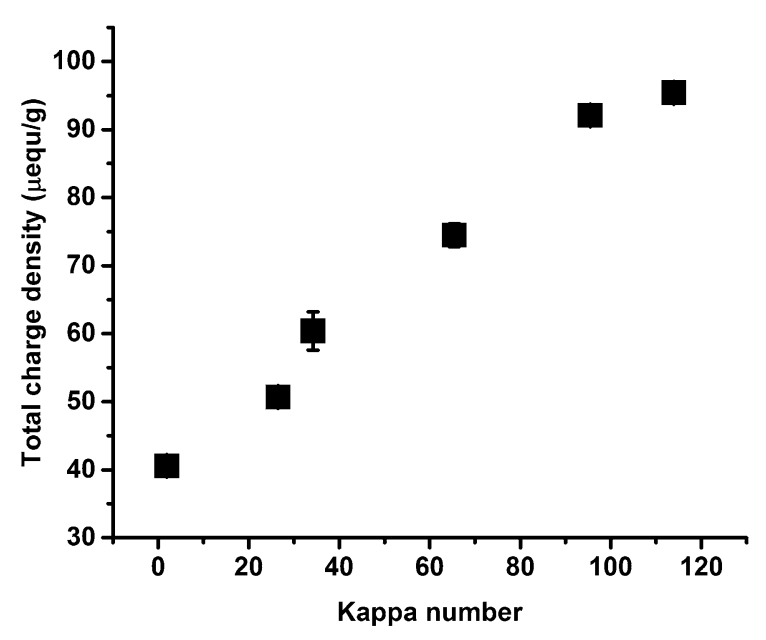

Fig. 3 Total charge density of kraft fibres versus Kappa number and lignin are gradually decreased through dissociation of xylan and lignin during pulping. Since there was no variation in the relative amount of xylan in the fibres, the main charge differences originated from variations in lignin content.

Figure 4 shows water retention value (WRV) of the pulp fibres against Kappa number. As Kappa number of fibres increased from 2 to 65 , WRV increased to a maximum. Possibly, swellable fibres with Kappa number 65 are suitable for homogenisation and mechanical disintegration into MFLC fibrils. A further increase in lignin content and Kappa number above 65 resulted in decreased WRV. Although lignin induces electrostatic charge to the fibres, it also "crosslinks" the fibrils in the cell wall. Thus, the swelling of fibres with higher Kappa number (lignin content) was

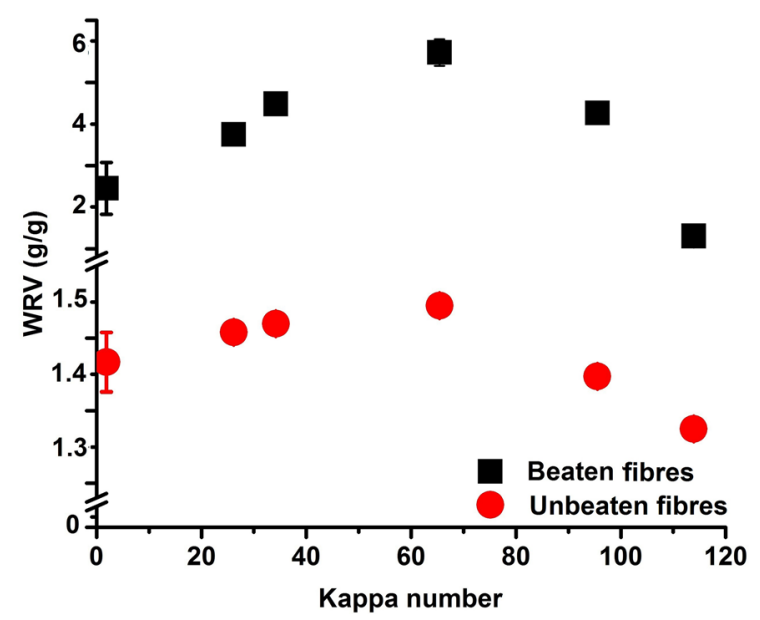

Fig. 4 Water retention value (WRV) of beaten and unbeaten kraft fibres versus their Kappa number (proportional to lignin content) 
Table 3 Physical and mechanical properties of micropaper (MP, from MFLC) and nanopaper (NP, from LCNF) structures

\begin{tabular}{lllllllll}
\hline Films & $\begin{array}{l}\text { Thickness } \\
(\mu \mathrm{m})\end{array}$ & $\begin{array}{l}\text { Basic } \\
\text { weight } \\
\left(\mathrm{g} / \mathrm{m}^{2}\right)\end{array}$ & $\begin{array}{l}\text { Apparent } \\
\text { density }\left(\mathrm{g} / \mathrm{cm}^{3}\right)\end{array}$ & $\begin{array}{l}\text { Apparent } \\
\text { porosity }(\%)\end{array}$ & $\begin{array}{l}\text { Water } \\
\text { contact } \\
\text { angle }\left({ }^{\circ}\right)\end{array}$ & $\begin{array}{l}\text { Tensile } \\
\text { strength } \\
(\mathrm{MPa})\end{array}$ & $\begin{array}{l}\text { Elastic } \\
\text { modulus } \\
(\mathrm{GPa})\end{array}$ & $\begin{array}{l}\text { Elongation } \\
\text { at break } \\
(\%)\end{array}$ \\
\hline MP-K2 & $22.8(1.2)$ & $31.6(0.8)$ & $1.39(0.08)$ & $7(5)$ & $38.8(1.3)$ & $226(19)$ & $13.6(0.7)$ & $6.4(0.9)$ \\
NP-K2 & $21.7(0.5)$ & $29.4(0.0)$ & $1.35(0.03)$ & $10(2)$ & $47.5(1.9)$ & $233(32)$ & $12.9(0.8)$ & $5.9(1.3)$ \\
MP-K65 & $20.9(1.0)$ & $29.4(1.1)$ & $1.41(0.09)$ & $6(6)$ & $84.0(2.2)$ & $237(17)$ & $13.7(0.3)$ & $5.2(0.6)$ \\
NP-K65 & $19.9(0.4)$ & $26.2(0.0)$ & $1.32(0.03)$ & $12(2)$ & $88.3(2.5)$ & $194(13)$ & $11.8(0.2)$ & $4.4(0.6)$ \\
MP-K114 & $24.9(1.3)$ & $29.1(0.3)$ & $1.17(0.06)$ & $22(4)$ & $72.8(1.5)$ & $174(11)$ & $10.8(0.6)$ & $4.4(0.7)$ \\
NP-K114 & $21.7(0.9)$ & $28.5(1.6)$ & $1.31(0.09)$ & $13(6)$ & $81.7(0.5)$ & $116(17)$ & $7.9(0.9)$ & $4.6(1.4)$ \\
\hline
\end{tabular}

K2, K65 and K114 refer to lignin content, see Table 1. Apparent porosity is calculated based on the assumed fibril density of $1.5 \mathrm{~g} /$ $\mathrm{cm}^{3}$. Values in parentheses are standard deviations

limited and showed lower values of WRV. The cell wall of fibres with low to moderate Kappa numbers (low to middle range lignin content), is delaminated and nanoporous as a result of delignification. However, due to hydrophilicity and repellence of charged carboxylic acid groups on the surfaces of fibres with moderate Kappa numbers, the fibres tend to swell and retain more water. Therefore, neither fibres with very low nor very high Kappa number had WRV as large as those with optimum Kappa number. The WRV-values of the beaten fibres showed a similar trend as the unbeaten fibres, although the extent of swelling was higher due to mechanical damage.

The aqueous suspensions of beaten kraft pulp fibres were subjected to mechanical homogenisation, in order to investigate the overall effect of lignin on the fibrillation of kraft fibres. The same fibrillation procedure was applied for all samples to enable comparison of the resulting MFLC suspensions. Pretreatments (e.g. oxidation) were not used.

The geometry of fibre fragments after homogenisation was studied using SEM and TEM. Figure 5 shows SEM images of typical large fibrils. The characteristics of branched fibrils are illustrated in images of MFLC-K2, MFLC-K65 and MFLC-K114. The large fibrils of MFLC-K2 and MFLC-K65 showed a width of $0.2-0.8 \mu \mathrm{m}$, while the width of MFLCK114 fibrils was typically $1-2 \mu \mathrm{m}$. The aspect ratio of fibrils was very large but could not be readily determined, see Fig. 5. Furthermore, in magnified images (see also electronic version), it was apparent that large fibrils were essentially "macrofibrils" and fibril aggregates from the cell wall (not yet disintegrated). The fibrils were branched at the ends, split into finer fibrils and again split into even finer fibrils. There were also occasional fibril entanglements, formed during sample preparation. Extremely fine fibrils are also present in the SEM images, but not clearly visible at the length scale of Fig. 5. The finer fibrils were separated by centrifugation and were characterized by TEM, see later section.

After centrifugation, the supernatant was separated to study morphology and weight fraction of the finest LCNF fibrils of each sample. The geometry of fibrils in the supernatant was quite similar for different samples. TEM images are presented in Fig. 6. Kinks from mechanical processing damage are apparent in the smallest fibrils, and the wider fibrils are branched, loose bundles of many small fibrils. There is no sign of lignin particles, which suggests the lignin is still molecularly mixed in the fibrils. LCNF-K114 (and LCNF-K65 to a comparatively lower extent) contained nano-particles, indicated with red arrows in Fig. 6 (different from pale unstained regions). These are probably lignin particles released from fibre cell wall during fibrillation, similar to the results of (Rojo et al. 2015) and (Jiang et al. 2019). The width of fibrils in LCNF, the supernatant of MFLC suspensions, were in the range of $2.5-70 \mathrm{~nm}$. The largest number of fibrils were fine fibrils $(2.5-5 \mathrm{~nm}$ thick) and some were fibril aggregates $(20-70 \mathrm{~nm})$. In Fig. 6, it is apparent that wide fibrils are bundles of fine fibrils originating from the cell wall structure, not yet disintegrated. The lengths of LCNF fibrils in the supernatant were at least several microns, similar to 


\section{MFLC-K2}
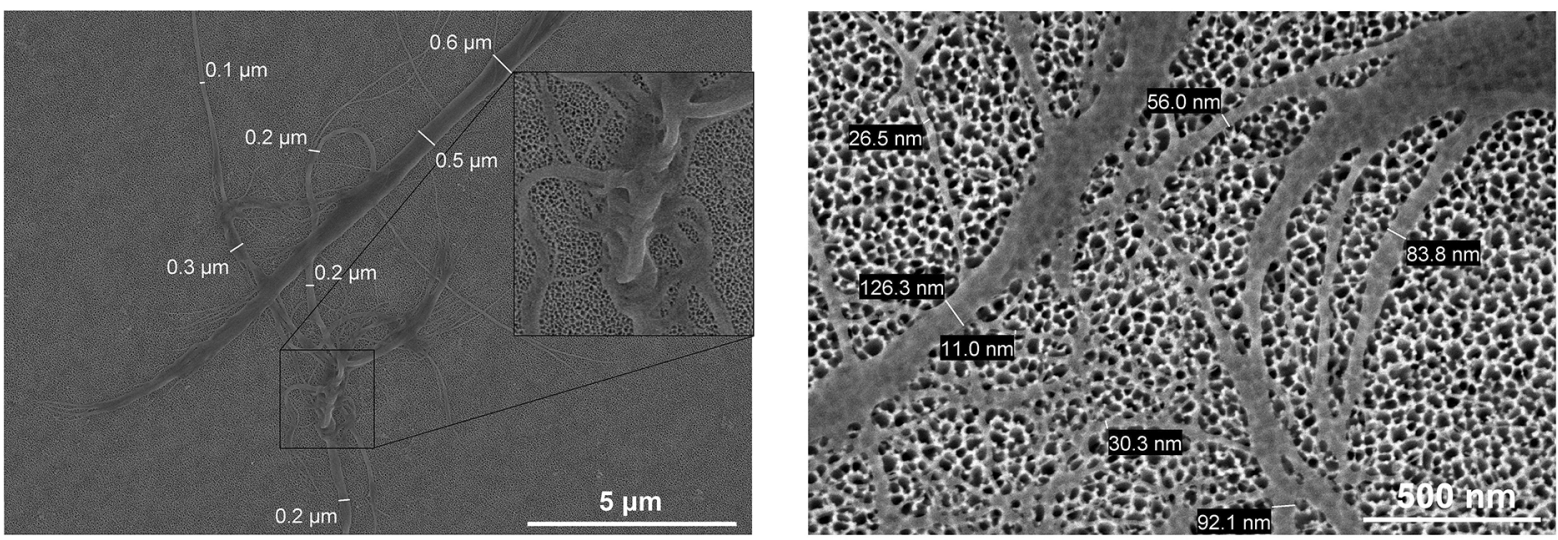

\section{MFLC-K65}
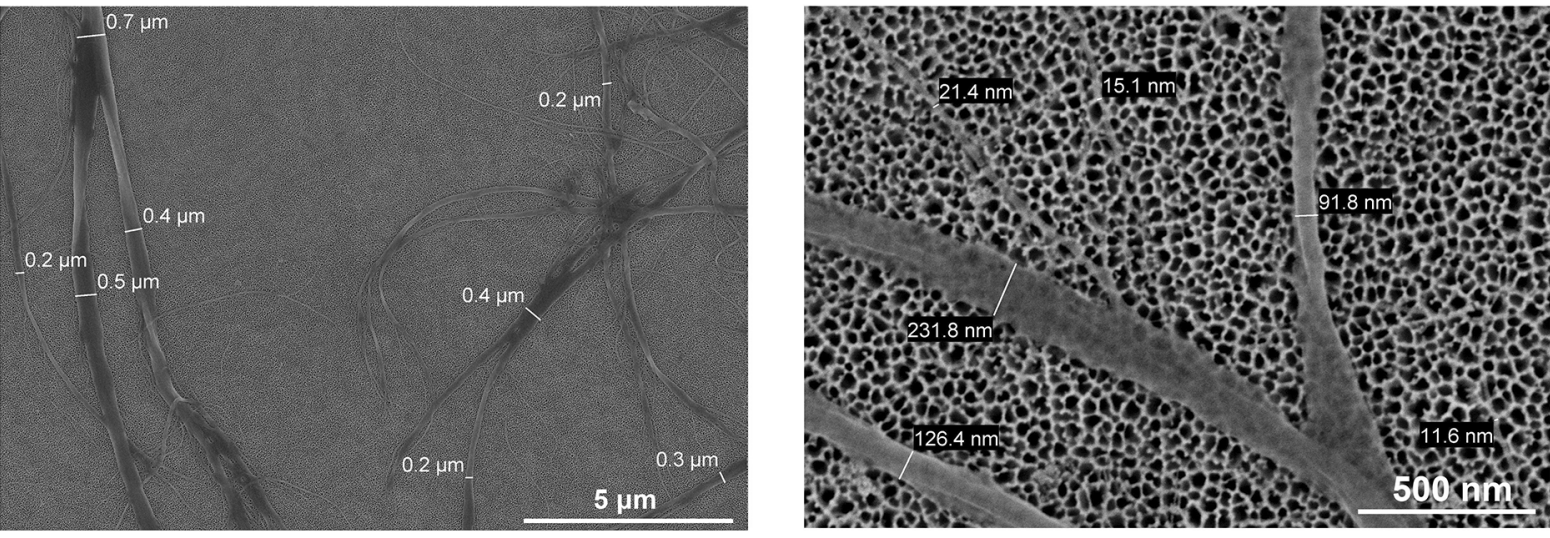

\section{MFLC-K114}

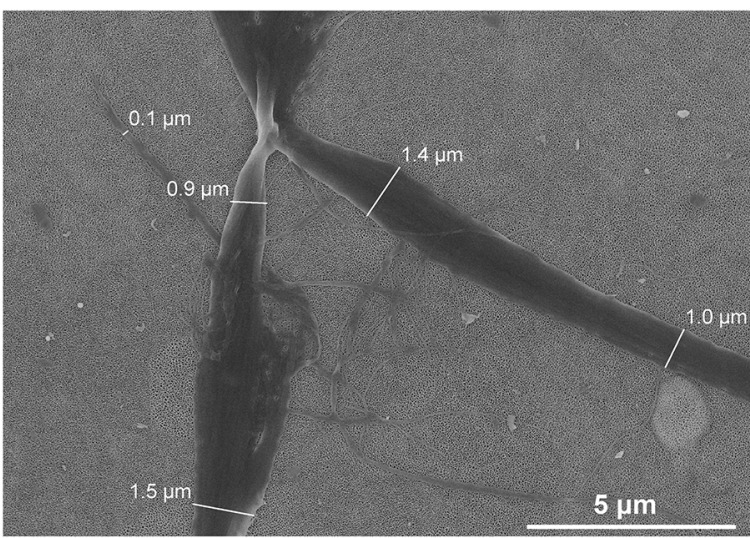

Fig. 5 SEM images of MFLC-K2, MFLC-K65 and MFLC$\mathrm{K} 114$ on a porous ceramic membrane. The right images are of the same samples at a higher magnification, showing branching

the estimated length of "microfibrils" in original wood (Atalla and Isogai 2010; Nishiyama 2009).

Although the fibril geometries appeared to be similar for different MFLC suspension supernatants,

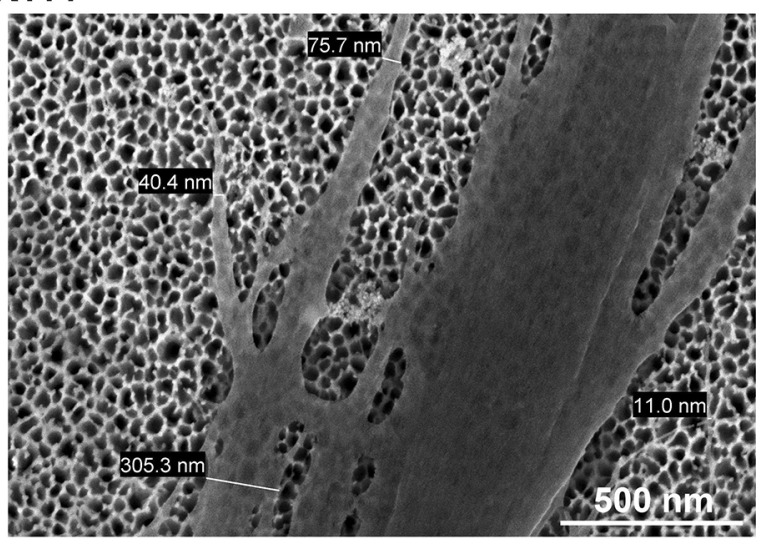

and finer fibrils. The focused rectangle in MFLC-K2 shows fibril entanglements. (High-resolution images are available in electronic version)

there was a difference in fibril weight fraction in supernatant. In previous studies (Moser et al. 2015; Naderi et al. 2015; Wågberg et al. 2008), the gravimetric yield of the cellulose nanofibrils was 
LCNF-K2
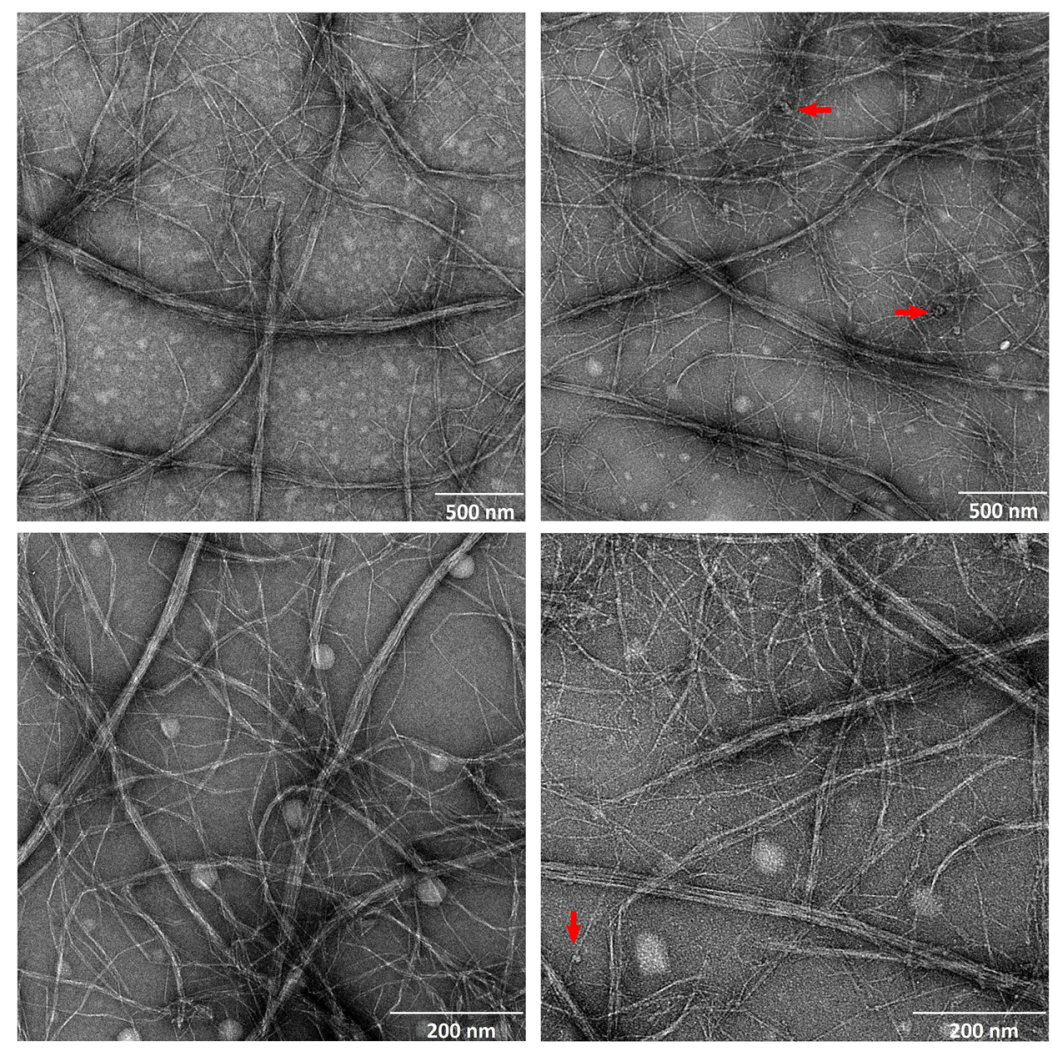

LCNF-K114
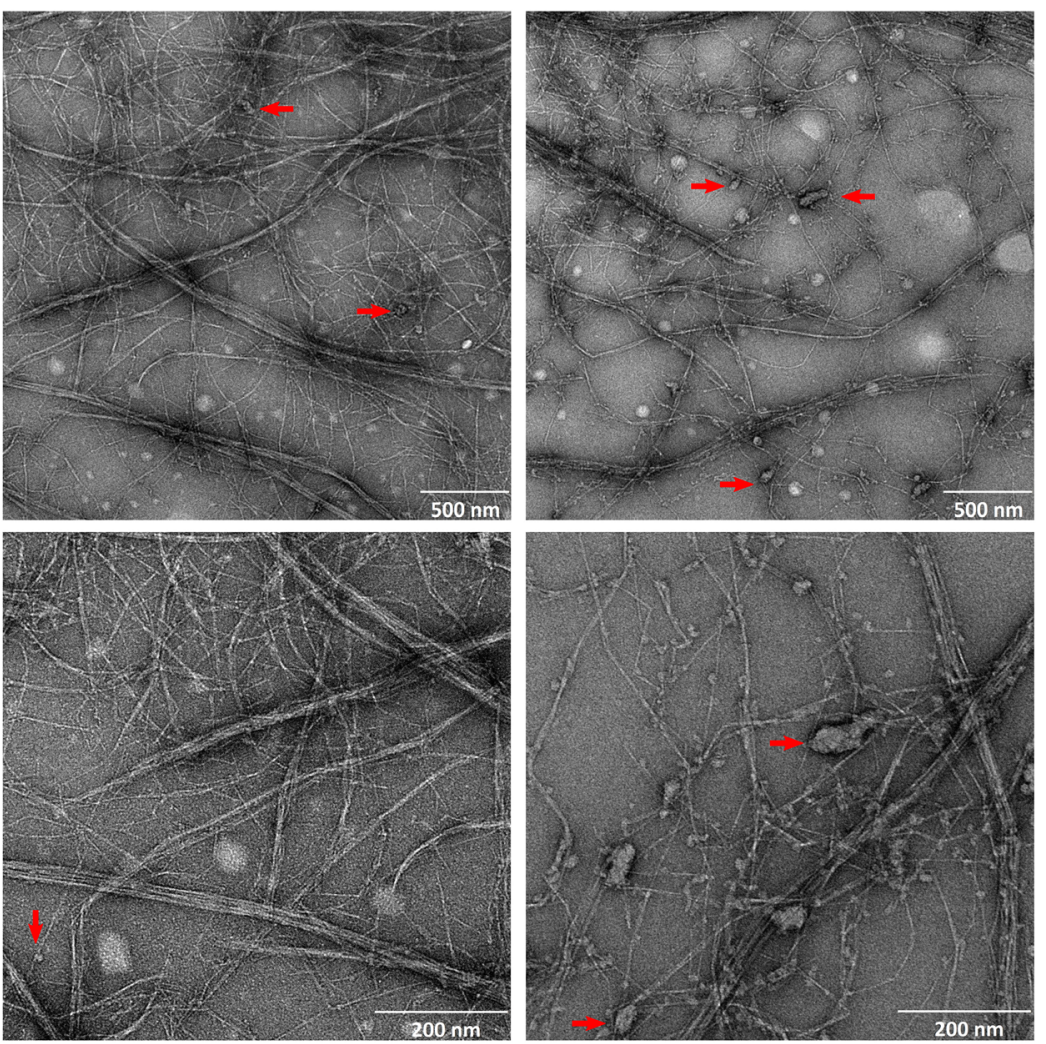

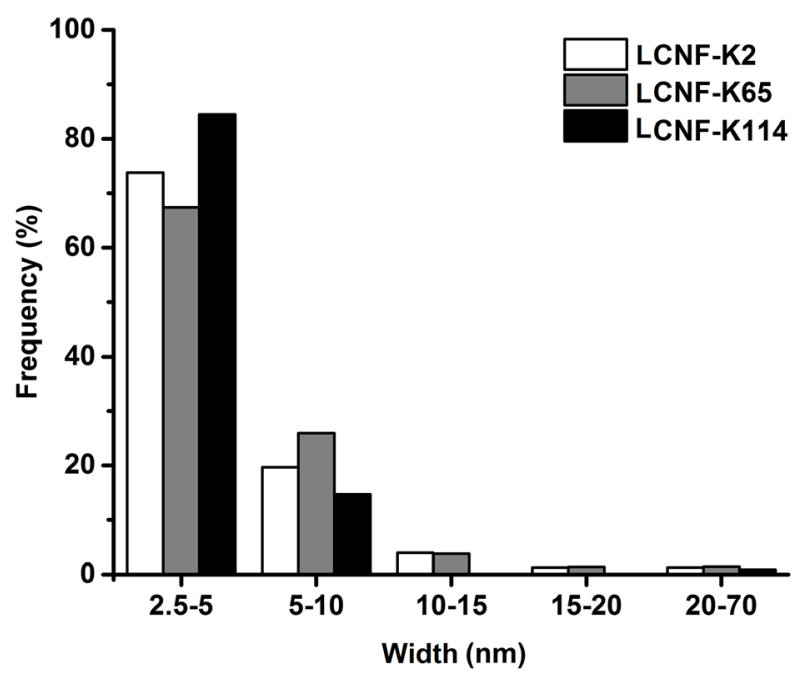

Fig. 6 TEM images of LCNF-K2, LCNF-K65 and LCNF-K114 (centrifugation supernatant of MFLC:s) and frequency of fibrils' widths

determined from centrifugation and used as an indication of fibrillation efficiency. For that reason, the ratio of supernatant to pre-centrifugation concentration was multiplied by the volume fraction of the
MFLC supernatant and this quantity was considered as the nanofibril yield. Figure 7 is the plot of nanofibril yield against Kappa number of the MFLC suspensions. MFLC suspensions with a middle range Kappa 


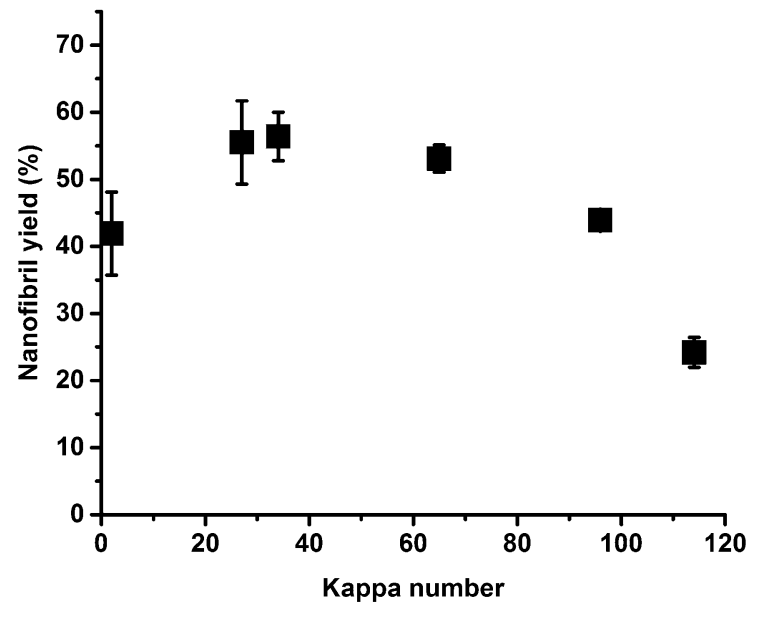

Fig. 7 Plot of nanofibril yield (mass fraction of supernatant), a measure of "ease of fibrillation", against Kappa number (lignin content measure) of MFLC suspensions

number (27-65) showed higher nanofibril yields than the bleached sample. The reason is that the charge content of fibres in middle range Kappa numbers facilitated fibrillation during homogenisation. At very high Kappa numbers ( $\geq 96$ ), the nanofibril yield of MFLC suspensions was lower since high lignin content impedes fibrillation. Continued mechanical homogenisation (7 further passes at 1500 bar) of MFLC-K114 reduced the width of largest fibrils (moslty to submicron), but did not improve nanofibril yield. Although high lignin content grades have higher charge which can facilitate fibrillation, the binding function of lignin is so strong that generation of very fine fibrils is prevented. Also, a previous study (Gunawardhana et al. 2018) showed that in the first homogenisation passes of thermomechanical pulp fines, generation of fine fibrils takes place. However, after 20 homogenisiation passes at 1000 bar, large fibre fragments were still predominant, because of the high lignin content.

Fibrillation of the sample with the highest Kappa number is difficult and results in the lowest nanofibril yield. SEM images of MFLC-K114 in Fig. 5 demonstrate larger fibrils compared to other samples. This observation is consistent with the low nanofibril yield of MFLC-K114, which means that the major weight fraction of MFLC-K114 is large fibrils, which sediment during centrifugation.

In order to estimate the potential of the present MFLC fibrils in materials applications, 6 nanopaper
Fig. 8 Upper: Photographs of micropapers (MP:s) and nanopapers (NP:s) from MFLC and LCNF grades with Kappa numbers of 2, 65 and 144 on a paper surface. Lower: optical transmittance and haze of MP:s and NP:s with thickness of ca $22 \mu \mathrm{m}$. See Table 3 for more information on micro-/nanopaper thickness and density

structures based on three different MFLC:s and three different LCNF:s (from supernatant after centrifugation) were prepared by a filtration procedure (Sehaqui et al. 2010). Note that for "micropapers" (MP:s), $100 \%$ of the MFLC suspensions were used and for "nanopapers" (NP:s) just the supernatant (LCNF) was used. Fibrils with Kappa numbers 2, 65 and 114 were investigated.

In Fig. 8, the nanopaper structures are presented with optical transmittance data. From photographs, the transparency is fairly high and logos underneath are apparent. In the blue light region, the measured transmittance of lignin-containing films is low, mainly due to the absorbance of chromophores present in lignin (Farooq et al. 2019; Li et al. 2016). Transmittance of NP:s is much higher than for MP:s because of lower scattering from small fibril aggregates. For the same reasons (lignin light absorption and scattering from large fibrils), NP-K2 and MP-K114 show the highest and the lowest transmittance, respectively. Haze is the ratio of diffused light (forward transmitted but scattered at large angle) to total light transmittance (Li et al. 2017). The haze is ca $80 \%$ for MP:s and ca $30 \%$ for NP:s. The lower haze is caused by smaller fibrils and in some case lower porosity (MP-K2 vs MP $\mathrm{K}-114$ ), which reduces haze-inducing scattering. Optical properties are thus highly sensitive to aggregated fibrils.

The physical and mechanical properties of micro/nanopaper structures (MP/NP) are presented in Table 3. Henriksson et al. (Henriksson et al. 2008) showed that an important contribution to yield strength and post-yield stress-strain behaviour of fibrous cellulosic films is interfibril bonding. Usually, improved fibril-fibril bonding results in better stress transfer at a given strain and enhanced stress level. MP-K2 and MP-K65 showed similarly high values of tensile strength and modulus, and similar density. MPK114 showed lower strength and modulus, and also lower apparent density (higher porosity) than other film structures, possibly due to the coarser constituent fibrils with high lignin content and weaker fibril-fibril 

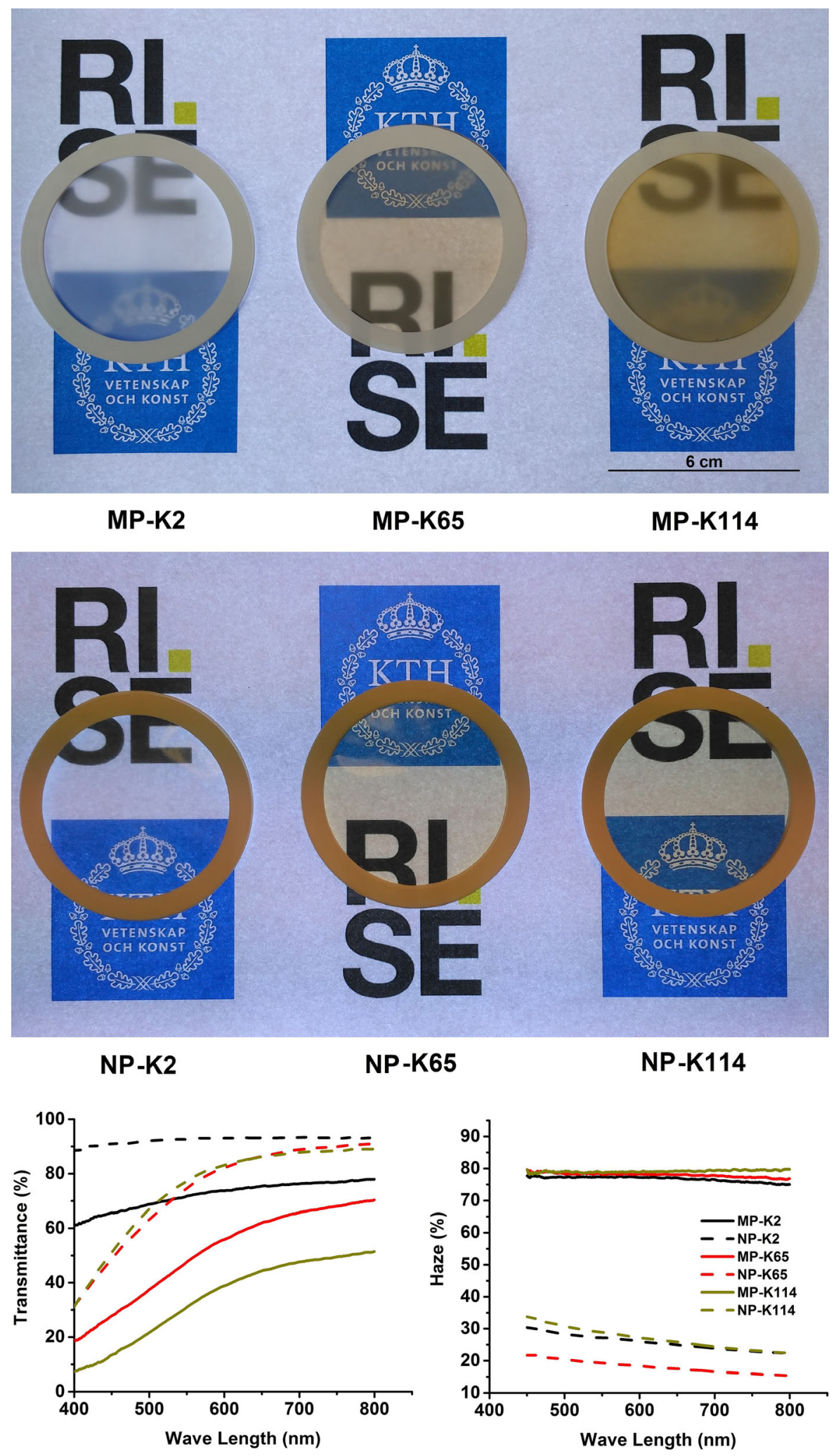
interaction. MFLC-K114 contained much more isolated lignin particles from fibrils, compared to MFLCK65 (see Fig. 6), which possibly compromised the fibril-fibril interaction. The nanopaper samples were, somewhat surprisingly, not stronger than micropapers despite finer structure. For NP-K2 and NP-K65, one reason is somewhat higher porosity. One may speculate that there is a higher amount of lignin particles in nanopaper compared to micropaper with the same Kappa number, which leads to weaker fibril-fibril interactions. There may be more isolated lignin particles present in the supernatant, which originate from disintegrated lignin from both sedimented and supernatant fibrils. Nevertheless, mechanical properties of NP-K65 and MP-K65 films were excellent, and further exploration of these grades was justified.

In Table 3, the variation in contact angles of micropaper and nanopaper with the same Kappa number, possibly stemmed from higher surface roughness in micropaper. Water contact angle of lignocellulosic films (e.g. films with Kappa numbers of 65 and 114) were larger than films from bleached samples, as reported in other studies (Herrera et al. 2018; Rojo et al. 2015). Although the ratio of polar to dispersive surface energies of lignin is smaller compared to cellulose (Notley and Norgren 2010), total surface energies of lignin and cellulose are similar (Eriksson et al. 2007; Notley and Norgren 2010). Considering Young's equation, the film-water interfacial energy was, therefore, the most influential factor for the contact angle. The interfacial surface energy with water was increased with increasing lignin content, since the molecular matching between the lignocellulosic film and water became less favourable.

MFLC-K65 is selected as the fibre in focus for further morphology studies since it has the highest lignin content of the candidate compositions, combined with high nanofibril yield (K27, K34, and K65), and the highest pulping yield out of K27, K34 and K65 (56.5\%, see Table 1). The corresponding micropaper structures from K65 showed very high mechanical properties and optical transmittance. The geometry of fibrils in MFLC-K65 was further investigated, based on a fractionation approach previously used for industrial MFC (Larsson et al. 2018).

The whole population of MFLC used for the micropaper films was fractionated, in order to quantify the coarsest fibre fragments, and estimate MFLC geometries. The coarse fractions were first screened with $20 \mu \mathrm{m}$ and $1-2 \mu \mathrm{m}$ meshes and the two fine fractions were separated by centrifugation. This method is a full fractionation method, in comparison with the simple centrifugation procedure used for yield measurements. The MFLC-K65 materials are further separated into F1, F2 and F3 fractions, along with the F4 supernatant fraction, see Fig. 1. SEM images of the four fractions of MFLC-K65 are presented in Fig. 9. There was a substantial difference in the fibril dimensions in each fraction. The coarsest fraction (F1) consisted of very large, branched fibre fragments, although the mass of this fraction was negligible. The second fraction (F2), was also very coarse, of very small mass fraction and fibrils showed a typical width of 1-2 $\mu \mathrm{m}$. The F2 fibrils showed substantial branching to finer fibrils. The fraction F3 consisted of fibrils with a typical width of $0.2-0.8 \mu \mathrm{m}$. F2 and F3 fibrils were "macrofibrils" with substantial branching. Macrofibrils are large fibril structures (Meyer and Misch 1937), which are the first to form during disintegration of fibres to fibrils (ChingaCarrasco 2011; Moser et al. 2019). The finest fraction, $\mathrm{F} 4$, consisted of fibrils with a width of less than ca. $70 \mathrm{~nm}$. These dimensions are also supported by the TEM images in Fig. 6. F4 fibrils consisted of "microfibrils" (fibril aggregates) and small fibrils similar to typical cellulose nanofibrils (CNF). The major mass fraction of MFLC-K65 is associated with F3 and F4 fractions. There was also mass loss due to rinsing of the screens with a large amount of water to remove trapped fine fibrils. The "Loss" fraction also consisted of F3 and F4, since those fractions most easily escape through the screens. This reveals that F3 and F4 together form about $96 \mathrm{wt} \%$ of MFLC-K65. It is indeed interesting that despite the presence of $\mathrm{F} 1$ and F2 fractions, the previously discussed micropaper structures based on MFLC-K65 show such excellent mechanical properties, see Table 3.

\section{Conclusions}

Microfibrillation of unbleached wood fibres is investigated and data are in support of an optimum lignin content (11\% lignin, Kappa 65, 70\% cellulose), where the nanofibril yield is maximized. The two competing mechanisms with increasing lignin content are increased charge, but also increased cellulose fibril binding effects from lignin. Previous MFLC studies are commonly 

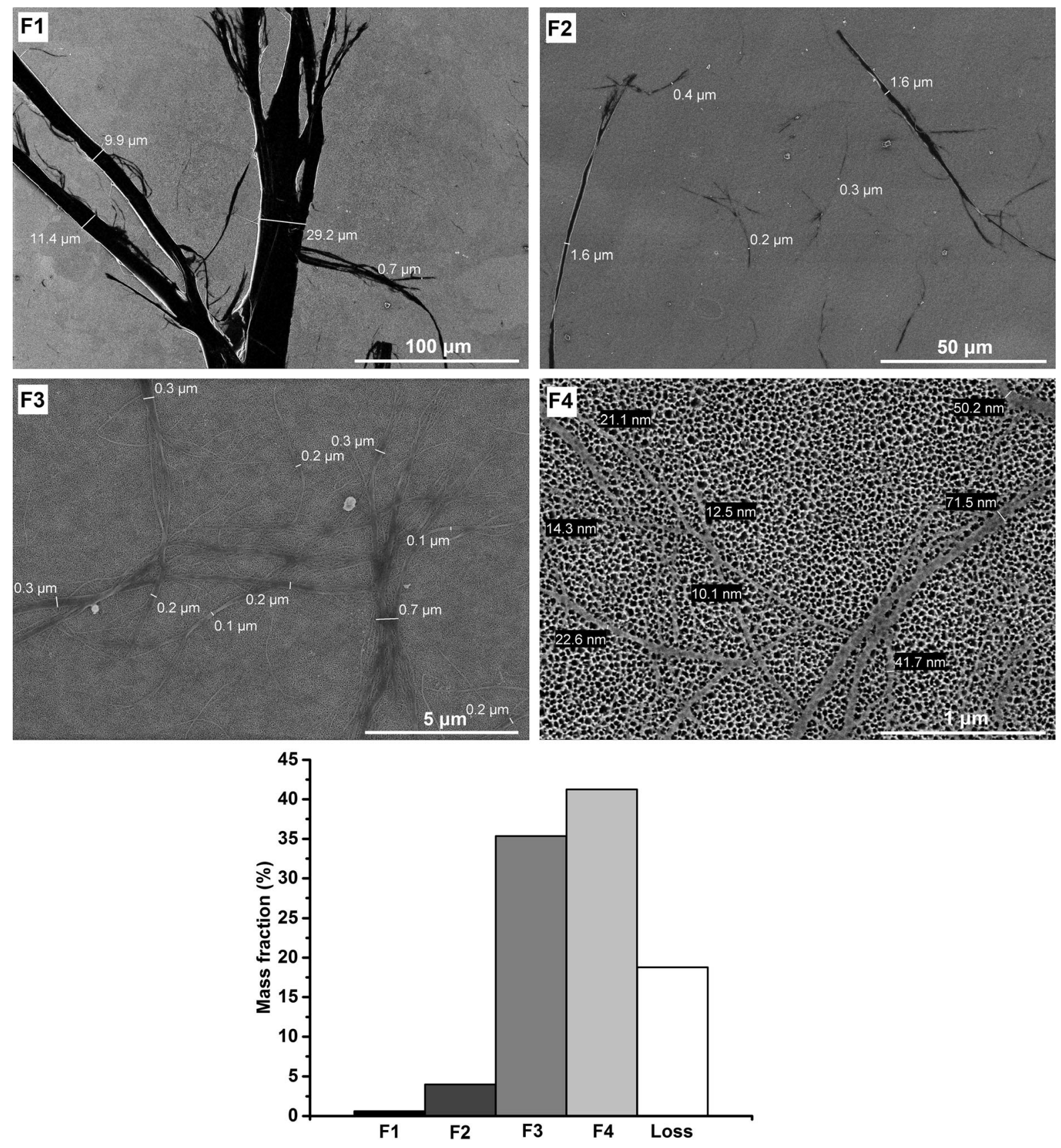

Fig. 9 SEM images and mass fractions of 4 different fractions of MFLC-K65 on a porous membrane: F1, coarsest fraction, F2, coarse fraction, F3, fine fraction and F4, finest fraction. Note the

either based on wood flour, specific pulp fibres, nonwood biomass or specific chemical treatment.

The present MFLC is of high technical relevance due to lignin content, no extra pre-treatment, scalable processing and use of high yield, unbleached kraft difference in scale bars for the different micrographs. The mass loss fraction is also presented, and primarily consists of F3 and F4 (High-resolution images are available in electronic version)

fibres available in industry. The high charge of the MFLC does not only facilitate fibrillation through cell wall swelling but also provides favourable colloidal stability to MFLC suspensions. A simple fractionation procedure shows that $95.5 \mathrm{wt} \%$ of homogenised 
Kappa 65 MFLC has a lateral dimension of 2.5-0.8 $\mu \mathrm{m}$. Nanopaper structures based on the whole MFLC population, including some large cell wall fragments, still showed good properties such as Young's modulus of $14 \mathrm{GPa}$ and ultimate strength $240 \mathrm{MPa}$, combined with a water wetting angle as high as $84-88^{\circ}$. MFLC from unbleached wood fibres is therefore a very interesting and practical building block for new materials, with different chemical characteristics than current industrial MFC from bleached fibres. MFLC should be further explored in the context of new materials, e.g. polymer matrix biocomposites and other fibrous materials from wood. From the point of view of quality control, nanopaper film data for optical transmittance and in particular haze, are much more sensitive to large cell wall fragment defects than data for ultimate strength.

Acknowledgments We would like to kindly acknowledge Lars Norberg for helping with Sprout-Waldron refining, Hui Chen for the help with optical transmittance, and Dr Per A. Larsson and Dr Göksu Cinar Ciftci for help with the fractionation set-up. Ann-Marie Runebjörk and Åsa Engström are also acknowledged for their support in the nanocellulose lab at RISE. The funding support of this work by the Swedish Foundation for Strategic Research, STFI association of interested parties and Wallenberg Wood Science Center, and open access funding provided by Royal Institute of Technology are gratefully acknowledged.

Open Access This article is licensed under a Creative Commons Attribution 4.0 International License, which permits use, sharing, adaptation, distribution and reproduction in any medium or format, as long as you give appropriate credit to the original author(s) and the source, provide a link to the Creative Commons licence, and indicate if changes were made. The images or other third party material in this article are included in the article's Creative Commons licence, unless indicated otherwise in a credit line to the material. If material is not included in the article's Creative Commons licence and your intended use is not permitted by statutory regulation or exceeds the permitted use, you will need to obtain permission directly from the copyright holder. To view a copy of this licence, visit http://creativecommons.org/licenses/by/4.0/.

Funding Funding was provided by Stiftelsen för Strategisk Forskning (Grant No. FID15-0115).

\section{References}

Abe K, Nakatsubo F, Yano H (2009) High-strength nanocomposite based on fibrillated chemi-thermomechanical pulp. Compos Sci Technol 69:2434-2437
Atalla RH, Isogai A (2010) Celluloses. In: Liu HW, Mander L (eds) Comprehensive natural products II. Elsevier, Oxford, pp 493-539

Berglund LA, Peijs T (2011) Cellulose biocomposites-from bulk moldings to nanostructured systems. MRS Bull 35:201-207

Chinga-Carrasco G (2011) Cellulose fibres, nanofibrils and microfibrils: the morphological sequence of MFC components from a plant physiology and fibre technology point of view. Nanoscale Res Lett 6:417

Dufresne A (2018) Cellulose nanomaterials as green nanoreinforcements for polymer nanocomposites. Philos Trans R Soc A Math Phys Eng Sci 376:20170040. https://doi.org/ 10.1098/rsta.2017.0040

Eriksson M, Notley SM, Wågberg L (2007) Cellulose thin films: degree of cellulose ordering and its influence on adhesion. Biomacromolecules 8:912-919

Farooq M, Zou T, Riviere G, Sipponen MH, Österberg M (2019) Strong, ductile, and waterproof cellulose nanofibril composite films with colloidal lignin particles. Biomacromolecules 20:693-704

Gellerstedt G, Lindfors EL (1987) Hydrophilic groups in lignin after oxygen bleaching. Tappi J 70:119-122

Gunawardhana T, Raj P, Varanasi S, Garnier G, Patti A, Batchelor W (2018) Development of cellulose nanofibre quality with mechanical energy: effect of starting material. In: Batchelor W, Söderberg D (eds) Advances in Pulp and Paper Research, Oxford 2017. FRC, Manchester, pp 139-158

Henriksson M, Berglund LA, Isaksson P, Lindström T, Nishino $\mathrm{T}$ (2008) Cellulose nanopaper structures of high toughness. Biomacromolecules 9:1579-1585

Herrera M, Thitiwutthisakul K, Yang X, Rujitanaroj P-o, Rojas R, Berglund L (2018) Preparation and evaluation of highlignin content cellulose nanofibrils from eucalyptus pulp. Cellulose 25:3121-3133

Herrick FW, Casebier RL, Hamilton JK, Sandberg KR (1983) Microfibrillated cellulose: morphology and accessibility. J Appl Polym Sci Appl Polym Symp 37:797-813

Herzele S, Veigel S, Liebner F, Zimmermann T, Gindl-Altmutter W (2016) Reinforcement of polycaprolactone with microfibrillated lignocellulose. Ind Crops Prod 93:302-308

Isogai A, Saito T, Fukuzumi H (2011) TEMPO-oxidized cellulose nanofibers. Nanoscale 3:71-85

Jiang Y, Liu X, Yang Q, Song X, Qin C, Wang S, Li K (2019) Effects of residual lignin on composition, structure and properties of mechanically defibrillated cellulose fibrils and films. Cellulose 26:1577-1593

Katz S, Liebergott N, Scallan AM (1981) A mechanism for the alkali strengthening of mechanical pulps. Tappi J 64:97-100

Klemm D, Kramer F, Moritz S, Lindström T, Ankerfors M, Gray D, Dorris A (2011) Nanocelluloses: a new family of naturebased materials. Angew Chem Int Ed 50:5438-5466

Klemm D, Cranston E, Fischer D, Gama M, Kedzior S, Kralisch D, Kramer F, Kondo T, Lindström T, Nietzsche S, PetzoldWelcke K, Rauchfuß F (2018) Nanocellulose as a natural source for groundbreaking applications in materials science: today's state. Mater Today 21:720-748

Larsson PA, Riazanova AV, Cinar Ciftci G, Rojas R, Øvrebø HH, Wågberg L, Berglund LA (2018) Towards optimised 
size distribution in commercial microfibrillated cellulose: a fractionation approach. Cellulose 26:1565-1575

Li Y, Fu Q, Yu S, Yan M, Berglund L (2016) Optically transparent wood from a nanoporous cellulosic template: combining functional and structural performance. Biomacromolecules 17:1358-1364

Li Y, Fu Q, Rojas R, Yan M, Lawoko M, Berglund L (2017) Lignin-retaining transparent wood. Chemsuschem 10:3445-3451

Lindström T (1992) Chemical factors affecting the behaviour of fibres during papermaking. Nord Pulp Pap Res J 4:181-192

Lindström T, Carlsson G (1982) The effect of chemical environment on fiber swelling. Svensk Papperstidning 85:R14$\mathrm{R} 20$

Lindström T, Aulin C, Naderi A, Ankerfors M (2014) Microfibrillated cellulose. Encyclopedia of Polymer Science and Technology. Wiley, Hoboken, pp 1-34

Lu H, Zhang L, Liu C, He Z, Zhou X, Ni Y (2018) A novel method to prepare lignocellulose nanofibrils directly from bamboo chips. Cellulose 25:7043-7051

Meyer KH, Misch L (1937) Positions des atomes dans le nouveau modèle spatial de la cellulose. Helv Chim Acta 20:232-244

Moon RJ, Martini A, Nairn J, Simonsen J, Youngblood J (2011) Cellulose nanomaterials review: structure, properties and nanocomposites. Chem Soc Rev 40:3941-3994

Moser C, Lindström ME, Henriksson G (2015) Toward industrially feasible methods for following the process of manufacturing cellulose nanofibers. BioResources 10:2360-2375

Moser C, Henriksson G, Lindström ME (2019) Structural aspects on the manufacturing of cellulose nanofibers from wood pulp fibers. BioResources 14:2269-2276

Naderi A, Lindström T, Pettersson T (2014) The state of carboxymethylated nanofibrils after homogenization-aided dilution from concentrated suspensions: a rheological perspective. Cellulose 21:2357-2368

Naderi A, Lindström T, Sundström J (2015) Repeated homogenization, a route for decreasing the energy consumption in the manufacturing process of carboxymethylated nanofibrillated cellulose? Cellulose 22:1147-1157

Nishiyama Y (2009) Structure and properties of the cellulose microfibril. J Wood Sci 55:241-249

Notley SM, Norgren M (2010) Surface energy and wettability of spin-coated thin films of lignin isolated from wood. Langmuir 26:5484-5490

Olszewska Eronen P, Johansson LS, Malho JM, Ankerfors M, Lindström T, Ruokolainen J, Laine J, Österberg M (2011) The behaviour of cationic nanofibrillar cellulose in aqueous media. Cellulose 18:1213

Osong SH, Norgren S, Engstrand P (2013) An approach to produce nano-ligno-cellulose from mechanical pulp fine materials. Nord Pulp Pap Res J 28:472-479

Peng Y, Nair SS, Chen H, Yan N, Cao J (2018) Effects of lignin content on mechanical and thermal properties of polypropylene composites reinforced with micro particles of spray dried cellulose nanofibrils. ACS Sustain Chem Eng 6:11078-11086

Rojo E, Peresin MS, Sampson WW, Hoeger IC, Vartiainen J, Laine J, Rojas OJ (2015) Comprehensive elucidation of the effect of residual lignin on the physical, barrier, mechanical and surface properties of nanocellulose films. Green Chem 17:1853-1866

Sarkanen KV, Ludwig CH (1971) Lignins: occurrence, formation, structure and reactions. Wiley, New York

Sehaqui H, Liu A, Zhou Q, Berglund LA (2010) Fast preparation procedure for large, flat cellulose and cellulose/inorganic nanopaper structures. Biomacromolecules 11:2195-2198

Sjöström E (1989) The origin of charge on cellulosic fibers. Nord Pulp Pap Res J 4:90-93

Sjöström E (1993) Wood polysaccharides. In: Wood chemistry: fundamentals and applications. Academic Press, San Diego

Spence KL, Venditti RA, Rojas OJ, Habibi Y, Pawlak JJ (2011) A comparative study of energy consumption and physical properties of microfibrillated cellulose produced by different processing methods. Cellulose 18:1097-1111

Turbak AF, Snyder FW, Sandberg KR (1983) Microfibrillated cellulose, a new cellulose product: properties, uses and commercial potential. J Appl Polym Sci Appl Polym Symp 37:815-827

Wågberg L, Decher G, Norgren M, Lindström T, Ankerfors M, Axnäs K (2008) The build-up of polyelectrolyte multilayers of microfibrillated cellulose and cationic polyelectrolytes. Langmuir 24:784-795

Walker JCF (2006) Primary wood processing: principles and practice. Springer, Dordrecht

Wei L, Agarwal UP, Matuana L, Sabo RC, Stark NM (2018) Performance of high lignin content cellulose nanocrystals in poly(lactic acid). Polymer 135:305-313

Wilson K, Goulding KH (1986) A biologist's guide to principles and techniques of practical biochemistry, 3rd edn. Edward Arnold, London

Yang X, Berthold F, Berglund LA (2018) Preserving cellulose structure: delignified wood fibers for paper structures of high strength and transparency. Biomacromolecules 19:3020-3029

Yousefi H, Faezipour M, Hedjazi S, Mousavi MM, Azusa Y, Heidari AH (2013) Comparative study of paper and nanopaper properties prepared from bacterial cellulose nanofibers and fibers/ground cellulose nanofibers of canola straw. Ind Crops Prod 43:732-737

Yousefi H, Azari V, Khazaeian A (2018) Direct mechanical production of wood nanofibers from raw wood microparticles with no chemical treatment. Ind Crops Prod 115:26-31

Publisher's Note Springer Nature remains neutral with regard to jurisdictional claims in published maps and institutional affiliations. 